\title{
PDAs and Shared Public Displays: Making Personal Information Public, and Public Information Personal
}

\author{
Saul Greenberg ${ }^{1}$, Michael Boyle ${ }^{1}$ and Jason Laberge ${ }^{2}$ \\ 'Department of Computer Science and 'Department of Psychology, University of Calgary, Calgary, Canada
}

\begin{abstract}
We are investigating how people move from individual to group work through the use of both personal digital assistants (PDAs) and a shared public display. Our scenario of this work covers the following activities. First, mobile individuals can create "personal" notes on their PDAs. Second, when individuals meet in real time, they can selectively "publicise" notes by moving them to a shared public display. Third, the group can manipulate personal and public items in real time through both PDAs and the shared public display, where the notes contained on both PDAs and public display are automatically synchronised. Finally, people leave a meeting with a common record of their activity. We describe our SharedNotes system that illustrates how people move through this scenario. We also highlight a variety of problematic design issues that result from having different devices and from having the system enforce a rigid distinction between personal and public information.
\end{abstract}

Keywords: Distributed groupware; Mobile computing; Personal digital assistants (PDAs); Single display groupware

\section{Introduction}

Recent years have seen the spread of computer technologies beyond the traditional desktop environment. Of particular interest to us is the advent of two new technologies and how they relate to Computer Supported Cooperative Work (CSCW). These are: personal digital assistants or PDAs, where people carry small handheld devices to store and retrieve personally relevant information; and single display groupware or SDG, where people gather face to face and work around a large shared public computer display.

What makes this choice of PDAs and SDG devices interesting to handheld CSCW is that both can be perceived as information appliances rather than as general purpose computers, where each device affords quite different personal and public acts. Norman [1,p53] defines an information appliance as:

\footnotetext{
...specialising in information: knowledge, facts, graphics, images, video or sound. An information appliance is designed to perform a specific activity. A distinguishing feature of information appliances is the ability to share information among themselves.
}

Today, PDAs are perceived as highly personal devices containing specialised personal information, while single display groupware are highly public devices containing specialised group information.
To become an information appliance, they need to share this information. The issue we are concerned with is how people conceptualise the exchange of information between these two quite different devices.

In essence, the problem is how people move their personal artefacts (created on their PDAs) into the public domain (manipulated on the SDG) and back again. Our general goal is to understand how people distinguish between these personal and public artefacts, its consequences on the design space for CSCW tools that run across multiple devices, and especially on how information is exchanged between devices.

To set the scene, we first describe what we mean by personal and public artefacts. We then present a scenario of use of SharedNotes, a combined PDA and SDG groupware system we designed that has a particular model of personal and public artefacts. We caution that SharedNotes is not a solution: it is a system designed to explore issues and provoke discussion. These are presented in the closing section.

\section{Personal and Public Artefacts}

Personal artefacts are things created, manipulated, and owned by one and only one person. Public artefacts differ, as they are created by cooperating 
group members, are considered owned by the group rather than any individual member, and can be viewed and manipulated by all.

Of course, these definitions are overly simplistic, as they just indicate two extremes in a spectrum. In everyday activity, people fluidly shift their artefacts from personal to public and the many gradations between. In a business context, for example, a person may prepare some personal notes, bring them to a meeting, and offer some or all of them for public consumption. Depending on the situation, others may copy these notes, add to them, and perhaps even take some of them away for their own personal use, thus completing the cycle from personal to public and back again. Another example is a group working around a shared visual surface, such as a large sheet of paper on a table top. Individuals may draw items close to themselves: while others are aware of this activity, these drawings are considered personal. At an opportune moment, the person may offer it to the group by drawing attention to it, thus making the drawing public [2]. At that point, the group may co-opt the drawing, where any member can add or modify it at will. Indeed, there are many ways people move artefacts between personal and public, as captured by words such as showing, copying, giving, exchanging, sharing, distributing, sub-dividing, and so on.

In these examples of everyday life, people's actions when shifting artefacts between personal and public are straightforward, often relying on criteria such as:

- who is actually holding the artefact

- how close the artefact is to others

- what is visible

- implicit social convention

- negotiated social convention.

A good example of how this works in practice is seen in Luff and Heath's study [3] of the way paperbased medical records support both synchronous and asynchronous collaboration between doctors, other professionals, and patients. The way the record is transported and made available to others makes it a resource as it is "passed between the hands of different professionals, is carried around the practice, and located in different parts of the office and clinic" [3,p306]. Particularly telling is their observation that "a doctor can position the record so as to invite the patient to view the materials; the text itself becoming the focal medium through which the talk is produced and interpreted
... the record can even be dismantled, letters removed, or reassembled in different ways; once again the separate mobility of the record's contents [is] exploited for various ways of acting and interacting with the patient" [3,p307]. Using these and other examples, Luff and Heath recommend that combinations of both mobile devices and fixed displays are needed so that "objects can be moved between the individual and private to the collaborative and public" [3,p312].

In contrast, most groupware systems either do not maintain people's distinctions between personal and public artefacts, or do so in ways that make the transition between the two awkward, heavyweight, or overly simplistic. To illustrate this point, we will look at a few ways that researchers and developers in mobile computing promote information sharing between devices.

Mobile computing encompasses the situation where people produce and share information in a mobile work setting, which seems to fit the work described here. In practice, however, the majority of mobile computing systems neglect the nuances between personal and public information exchange.

1. Mobile devices can serve as a means for people to augment real-time personal communication. Researchers at Carnegie Mellon University, for example, designed a system that allows a remote person: to see (via video) what a field worker sees; and to share documentation $[4,5]$. While sharing does occur, there is no permanent transfer of information.

2. The mobile device lets people download communal information (such as that held in a database), modify it, and upload it again. These systems emphasise the public aspect, as all "personal" work will feed right back into the database.

3. The mobile device lets people gather personal information 'in the field', which is then uploaded into a communal database. An example applied to CSCW is NotePals, where participants at (say) a conference session write meeting notes on their PDAs. These are later shared with other meeting participants by synchronising with a shared note repository: notes can then be viewed using a desktop-based web browser [6]. Note that this method provides only a one-way information flow from personal to public.

4. Another approach is to give the mobile person access to one's workstation environment. PalmVNC, for example, lets the mobile 
PDA user connect to and view a portion of their workstation screen [7]. Other systems migrate or "teleport" a person's working environment to nearby workstations. by migratory applications $[8,9]$.

5. Some mobile systems synchronise personal information across devices. For example, the 3Com Palm Pilot HotSync Manager replicates information held in equivalent applications across both PDA and desktop computers [10].

6. More powerful are synchronisation systems that lets people synchronise both personal and public information across devices. With Lotus EasySync, for example, information within Palm Pilot applications (addresses, memos, email, calendars) are synchronised with a Lotus Notes database [11]. Because of the power of Lotus Notes, the database can: contain any mix of personal and public information; maintain people's identities actoss transactions; and enable different access controls. Still, the model in EasySync is that the information in the database and the PDA applications should be the same. When conflicts occur (due perhaps to multiple people changing the same data field), it is up to the user to resolve them into a single entry that contains the "correct" information.

7. Much research into mobile computing considers it as a technology. Examples include how occasional and permanent wireless data communication can be supported (e.g. [12]); new methods for handling user input (e.g. NaviPoint [13]); and new forms of portable devices. While technical innovations will impact how one treats personal and public information, they do not directly address the issue.

8. Techniques for information sharing across devices delegate the notion of public versus. private into low-level interaction techniques. One approach is Pick and Drop [14], where people can "pick up" the artefact from one penbased device and "drop it" onto another penbased device. This technique is excellent for implementing simple models of information exchange between devices, such as copying and moving.

Our specific research interest is to understand the nuances of how people shift artefacts between the personal and public, and the resulting design implications to CSCW systems that includes PDAs and SDG. To explore these nuances, we built a system called SharedNotes, described next.

\section{Shared Notes: SCENARIO Of Use}

We used the GroupKit groupware toolkit [15] to implement a groupware system called SharedNotes. Using SharedNotes, people can create and manipulate both personal and public notes between three types of devices: a PDA, a personal workstation, and a public SDG display. In particular, our PDAs are 3COM Palm Pilots (one for each person), our workstations are conventional PCs (typically situated in one's office), while our public display is a Smart Technologies [16] Rear Projection Smart Board 720 (a 72" diagonal, pen-based and rearprojected computer display) situated in a small meeting area. SharedNotes also allows distributed real-time meetings, where remote participants can connect to the session from their workstations.

We begin with a scenario of activity that illustrates how we envisage the use of our SharedNotes system. The scenario will illustrate the following points.

- Personal work. Individuals can create, rank, and annotate personal notes created on their PDAs.

- Moving from personal to public. Individuals can selectively publicise notes: when the group meets in real time, they are automatically replicated to a shared public display.

- The public arena. The group can create and manipulate both personal and public items in real time through both PDAs and the shared public display. Notes contained on both PDAs and public display are automatically synchronised.

- Between meetings. People leave with a common meeting record on their PDA. The record is not static, as they can continue working with it (as in the first point), and can then bring new notes back to the next meeting (as in points 2 and 3 ).

Our scenario describes how a few people who are setting up a usability laboratory explore their equipment requirements. We will imagine that this began through an email exchange, where one person suggests to others that each think about what equipment is needed, and that all would meet to discuss these items in a meeting on the following day. We will take the perspective of Michael, one of the team members.

\subsection{Personal work}

While taking the bus home, Michael starts thinking about some of the equipment needed. He pulls 


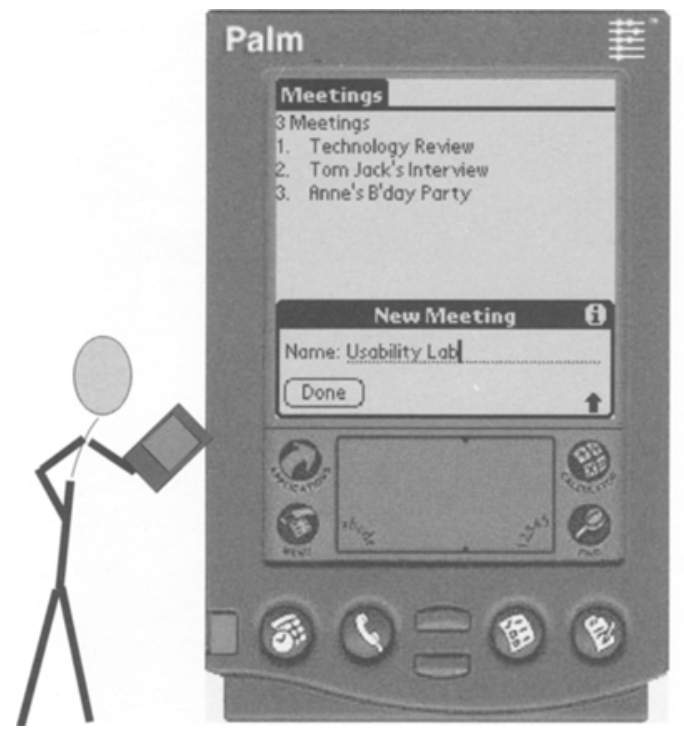

Fig. 1. Creating/selecting a meeting.

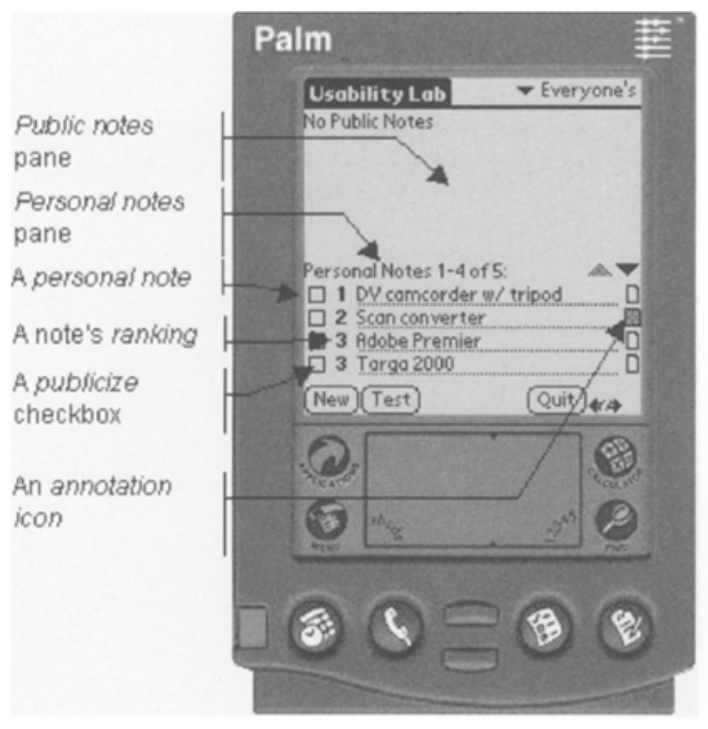

Fig. 2. The personal/public space.

out his PDA and creates a new meeting called Usability Lab which will contain his - and eventually all other people's - notes on the topic (Fig. 1). He enters the meeting and starts listing some equipment he would like to see. These appear as items in the "Personal Notes" pane of his display (Fig. 2, lower half). He also gives each item a rank, from 1 (high priority) to 3 (low priority) (Fig. 2, lower left). He then adds an annotation to one of his notes by tapping an annotation icon (Fig. 2, right side) and filling in the pop-up Note

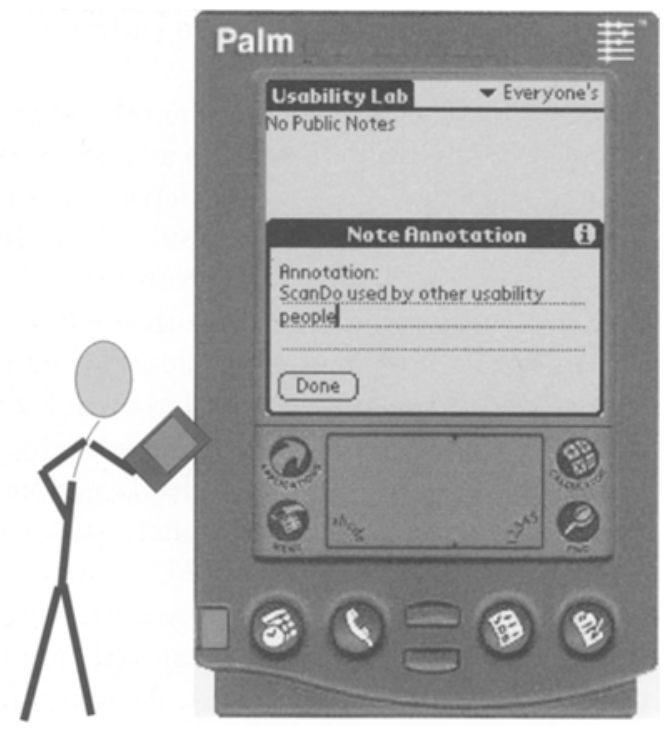

Fig. 3. The annotation dialogue.

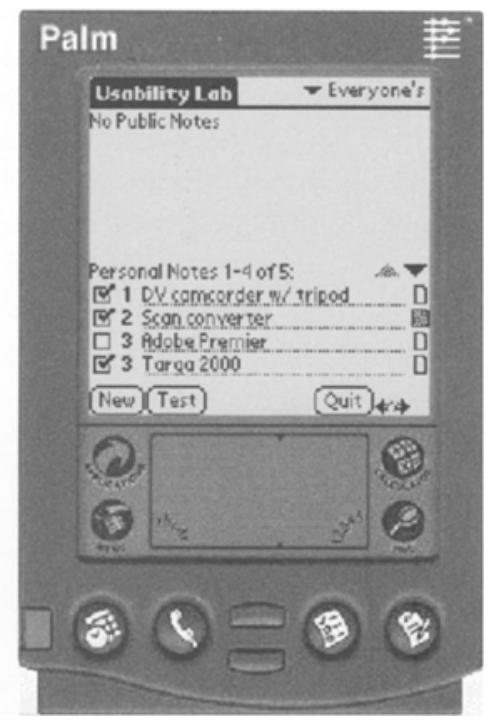

Fig. 4. Pending public notes.

Annotation dialogue (Fig. 3). (Notes with attached annotations are indicated by a darker annotation icon.) Finally, he decides that he would like to tell the group about some, but not all of the equip-ment he has listed. He does this by tapping the "publicise" checkbox next to the relevant notes, which causes them to be publicised to the rest of the group when they next meet (Fig. 4). Over the course of the evening, he sporadically creates new notes, modifies existing ones, and adds annotations as ideas come to him. 


\subsection{Moving from personal to public}

The following day, Michael and the others meet in their laboratory, which includes a large public display and attached PC. Because Michael happens to be the first one there, he turns on the display and readies the version of SharedNotes that acts as SDG and that communicates with the PDAs. He then seats his PDA on a cradle attached to the computer (in the future, this could occur automatically through wireless technology), which immediately propagates all of Michael's personal notes that have been marked as publicise onto the public display (Fig. 5). Unlike the PDA interface, the public display actually behaves as a structured drawing editor: text notes can be moved around and drawing marks can be added. As notes are publicised, they are moved from the personal to public pane on Michael's PDA. For example, the snapshot in Fig. 6 shows the personal and public display being synchronised, and how the publicised notes have moved onto the upper public pane.

The other people start coming into the room, and connect as well. As they do so, the personal notes they had checked for publication are also propagated to the public display (Fig. 7). Conversely, all public notes generated by the group now appear on each person's PDA in the Public Pane (Fig. 8). In Fig. 8, we see that Michael's PDA now containing other people's notes (e.g., there are now nine public notes) that were checked as publicise by other people on their PDAs. If Michael wanted to, he could choose via a menu option to see all publicised notes (as in Fig. 8, indicated by the "Everyone" menu button in the upper right corner), only his own publicised notes, or only other people's notes.

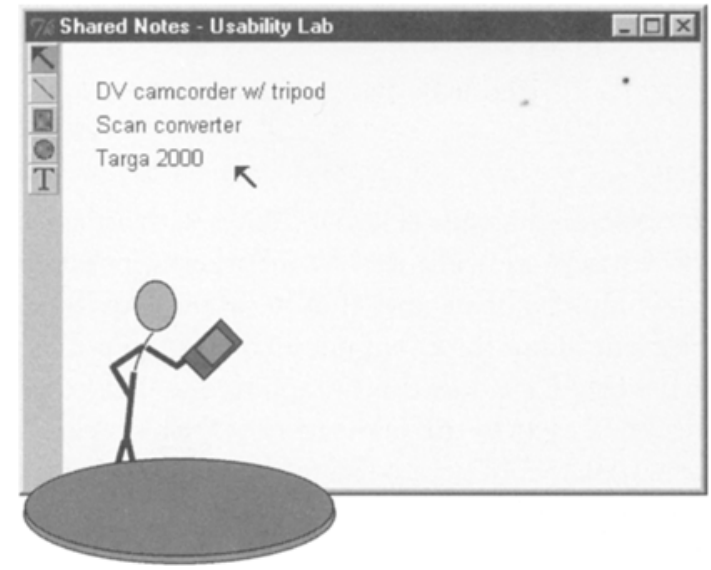

Fig. 5. The public display after Michael has connected.

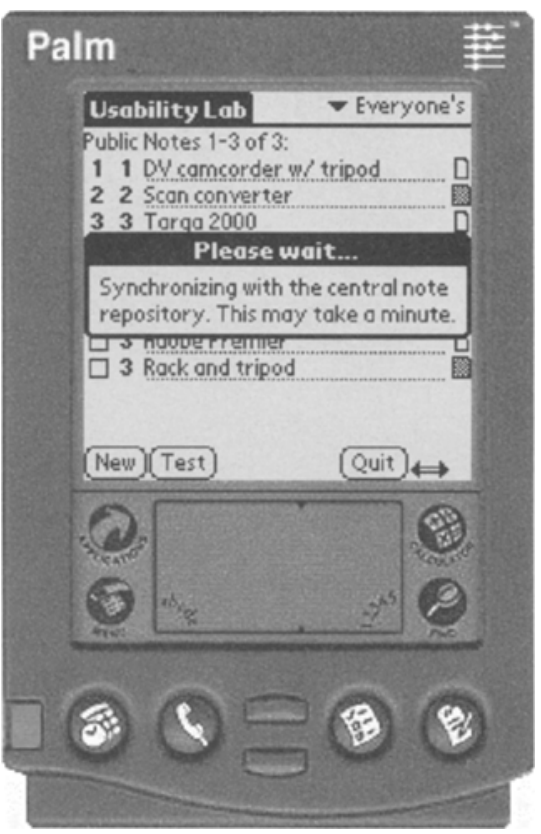

Fig. 6. The Pilot display during connection.

Shared Notes - Usability Lab $\square|\square| x$

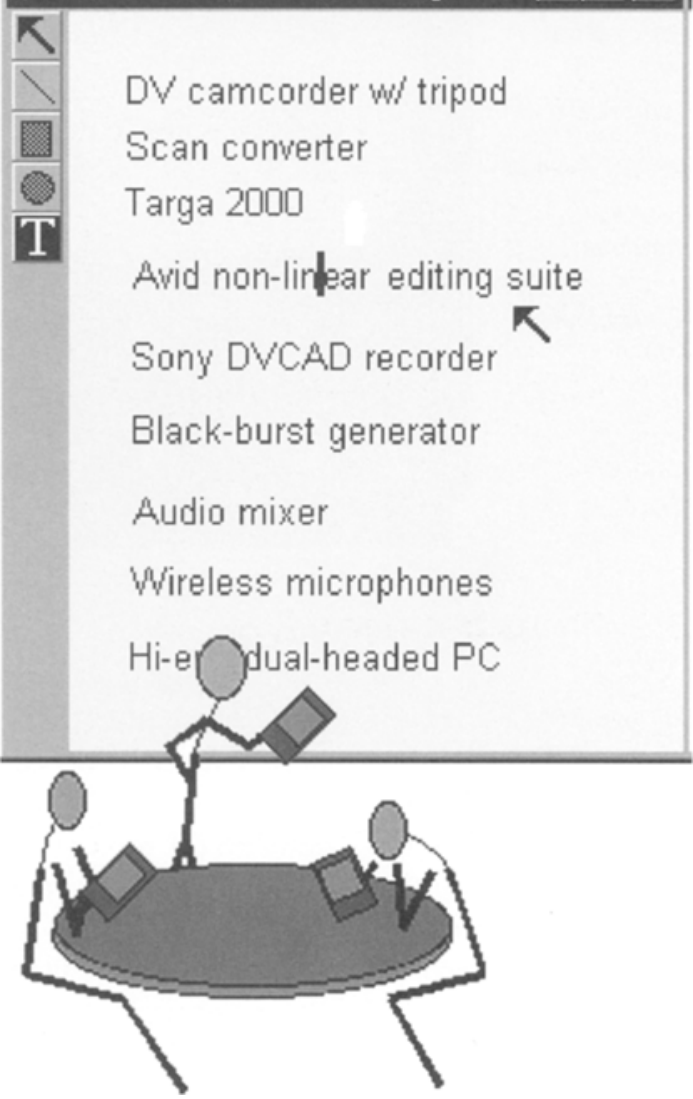

Fig. 7. The public display showing public notes. 


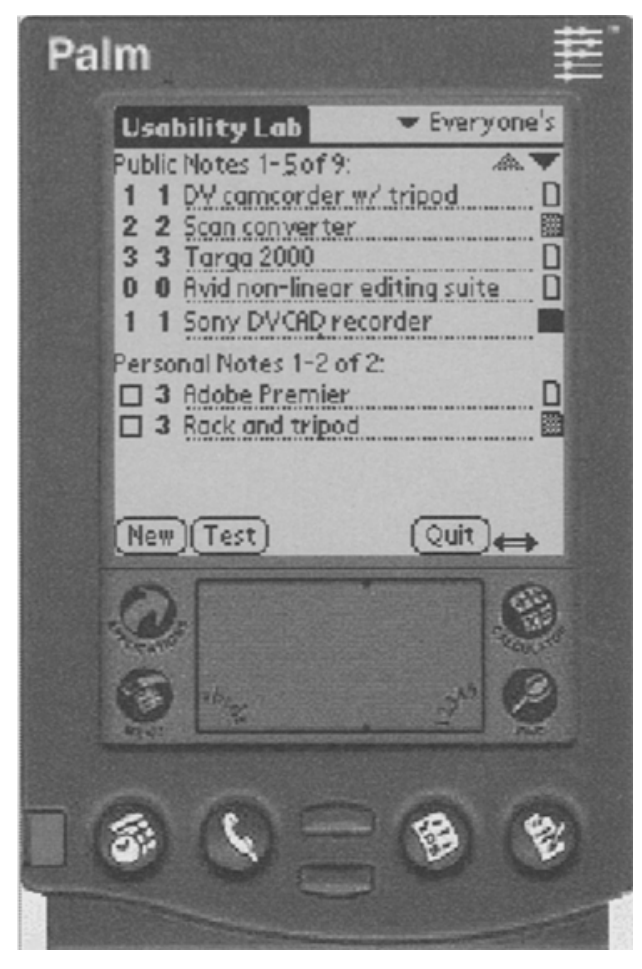

Fig. 8. The Pilot display showing public notes.

Finally, people can also connect to the public display remotely using their PDA attached to their personal workstation. When they connect to the session, they see both a replicated copy of the public display (Fig. 9, top half) and an interface similar to the PDA system on their own workstation (Fig. 9, bottom). This allows them to manipulate personal information via either the PDA or its workstation equivalent, and public information via the public display replica. Similarly, public displays can be linked across meeting rooms.

\subsection{The public arena}

At this point, people can discuss these notes and individuals can manipulate them via the public display and their PDAs. However, the PDAs and public screen enable different features and powers.

On the public display, people can create new text items and edit older ones directly (e.g. a new note titled "Studio monitor" is being added at the bottom of Fig. 10), and these will propagate down to the PDAs (Fig. 11, last item in the public pane). These text items behave just like the other drawing objects, and can be repositioned, deleted, selected, and so on, as illustrated in the differences between
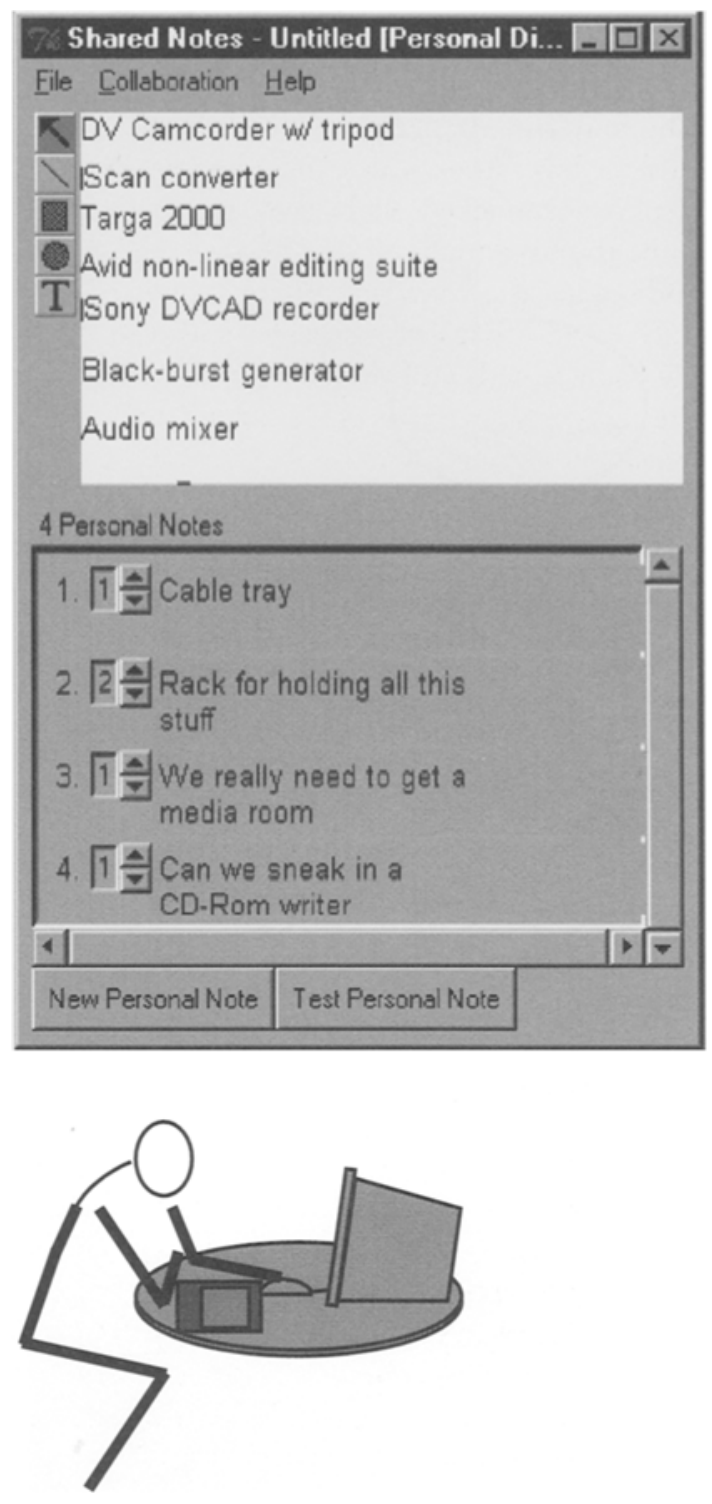

Fig. 9. The public display showing public notes.

Figs 7 and 10. Participants can also draw on the public display e.g. the box and circle in Fig. 10. Annotations are revealed by selecting an item (the pop-up window in Fig. 10). While individual's personal annotations cannot be edited on the public display, a new "Public" annotation can be added and edited (in this case, the public display is named "GroupLab".

On the PDA side, Michael can continue to create personal notes and publicise them at will. By selecting the annotation indicator for any note in the Public pane, he can view annotations made by others, and can edit his own annotation (Fig. 12). He can change his own ranking of that note, 


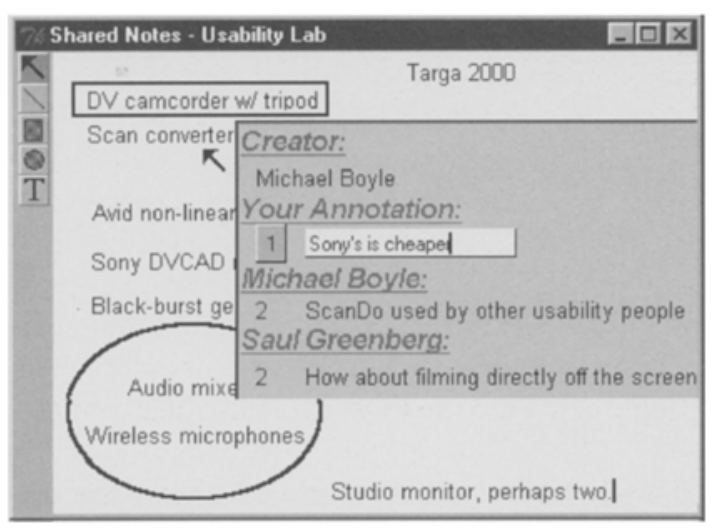

Fig. 10. Viewing all public notes and an annotation.

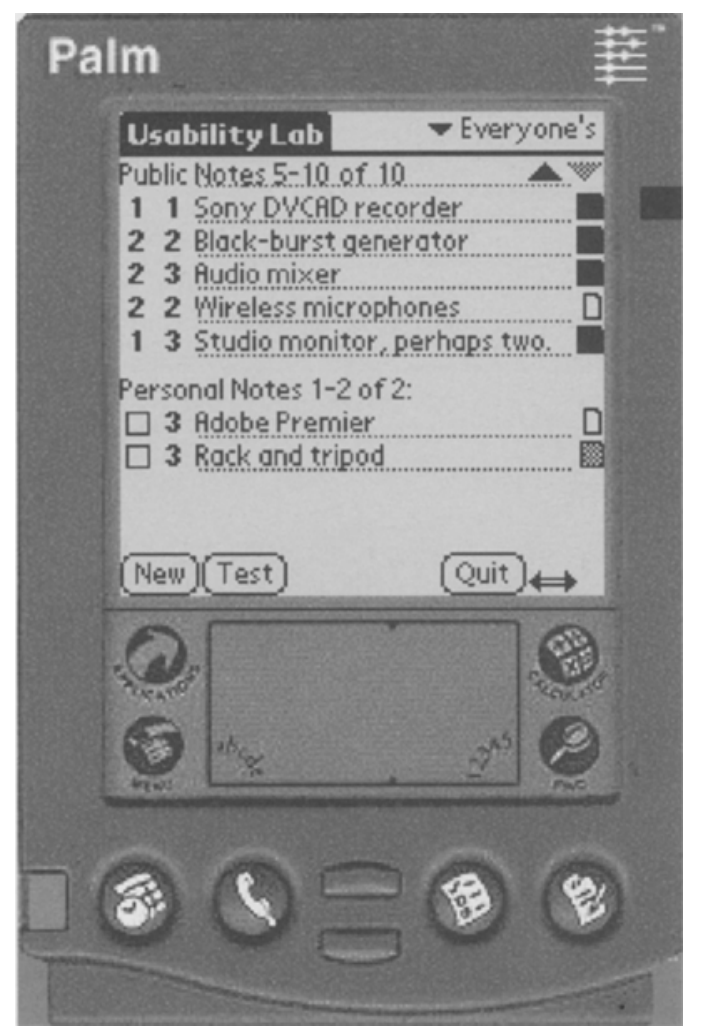

Fig. 11. The updated public note.

which will be reflected back in the average ranking score. However, he cannot change the contents of the actual text note for reasons that will be discussed later.

During this meeting, people may move between connected and disconnected work, simply by moving their Palm Pilots in and out of the cradle. The contents are resynchronised when connections are made.

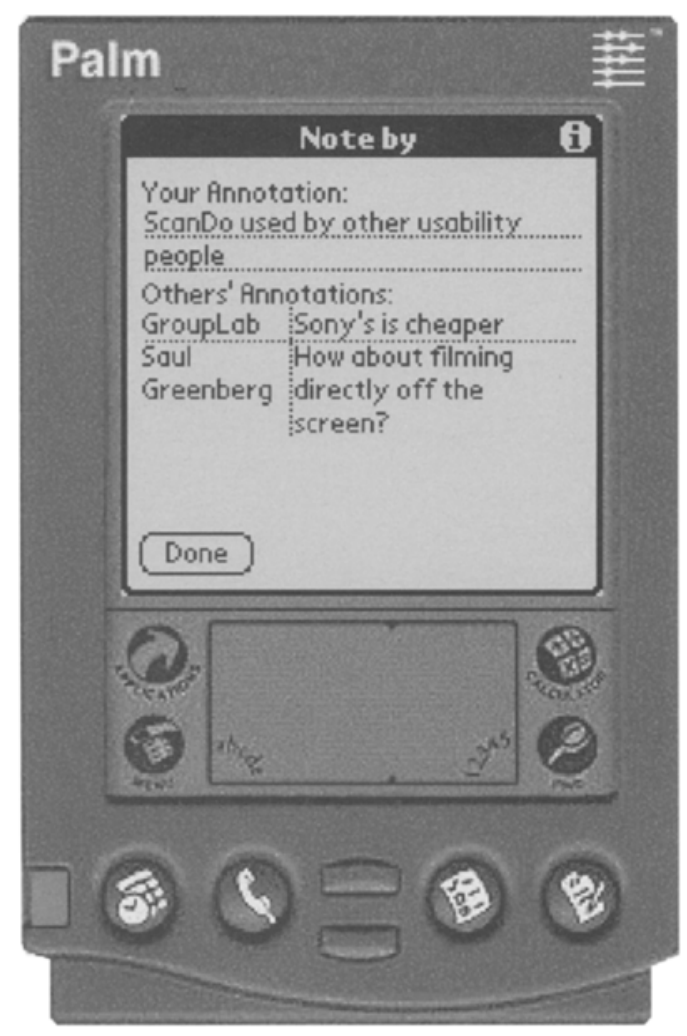

Fig. 12. Public annotations.

\subsection{Between meetings}

At the meeting's end, the session is saved. The public display maintains a persistent version of all the work done so far (e.g. Fig. 13 shows the final view of the public display). Michael (and all the others) walk away with a textual record of the meeting notes on their PDAs. However, this is not the end. Over the next few days, Michael and the others can add and edit new personal notes, and can further annotate or re-rank public notes on their personal PDAs. Alternately, one or more of the group members can work on the public display as needed. Whenever connections are made, the information is automatically synchronised and propagated as required.

\section{Discussion}

While the scenario is plausible (and the interface perhaps convincing), there are several oddities in the system itself. In particular, we intentionally made design decisions that took a somewhat extreme stance on the way people had to perceive 


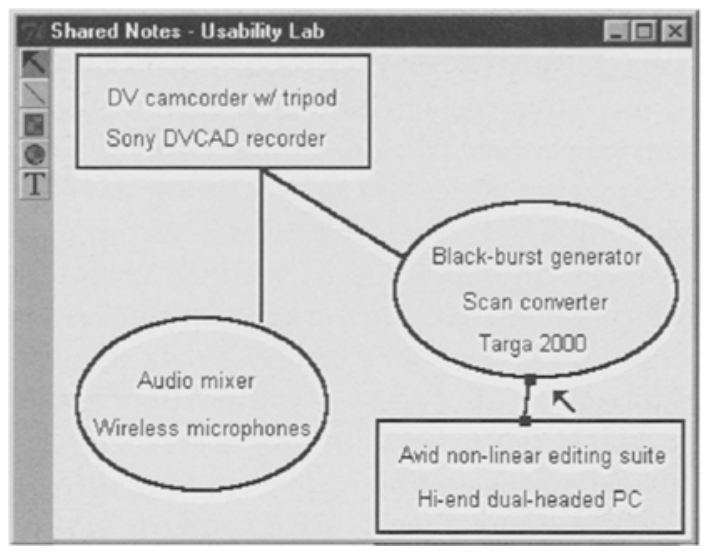

Fig. 13. The public display at the end of this meeting.

and manipulate personal and public artefacts ${ }^{1}$. We did this to draw out design nuances and resulting issues, rather than hide them.

The discussion below illustrates some of these issues, and is drawn from our own reflections on the design, from our own experiences using SharedNotes, and from watching others use it.

\subsection{Design issues raised by the differences between devices.}

The first set of design issues arise because the various devices used in our scenario - PDAs, large public displays, and workstations - are quite different.

\section{Should the PDA serve as an equal partner to other groupware appliances?}

A variety of researchers have used PDAs merely as input devices to SDG, where users can control and manipulate the information visible on the large display. For example, with the Remote Commander a person uses the PDA to control conventional applications such as PowerPoint [17]. Similarly, a team can draw simultaneously within PebblesDraw, a SDG system that captures input streams from individual PDAs [17]. While obviously useful in some situations, treating the PDA as only an input device makes it a subordinate groupware appliance: it does not maintain any strong notion of the meeting and its artefacts

${ }^{1}$ Our design stance is extreme only after the fact. During implementation, we faced many design choices. When it was unclear which choice would work best, we took the more provocative alternative in order to see what would happen. beyond the ability to manipulate them during the course of the meeting.

In contrast, our view is that the PDA is a very powerful personal device that can be applied to groupware: although it has different powers from the public display, it should be an equal rather than subordinate partner. In particular, as an equal partner it can maintain a personal view of the meeting activities. We saw this in SharedNotes, where people could use the PDA to create their own personal notes, to propagate selected ones to the Shared Display, to gather and take away the collected notes of the group, and to continue their work afterwards. This view is closer to Norman's [1] information appliance idea, where the PDA is treated as a specialised device that shares information with other specialised devices.

\section{Should each device be treated as a different entity enabling different acts?}

We could have designed the PDA and public screen to have identical interfaces, e.g. where the public display would mimic the PDA screen, or where the PDA would present a portion of the public display (as done by PalmVNC, [7]). However, these devices are quite different - display size, input speed and accuracy, portability, personal availability, processing capabilities, etc., - and we believe that the types of things one would do with each device demands a different set of powers and interface features. Consequently, we felt that each device should be treated as a different entity enabling different but complementary acts within a groupware setting.

In our design of SharedNotes, we decided to make the software running on each device different in several ways, where the PDA would be better at personal note creation, and the public display better for promoting group discussion. First, we wanted to retain the notion that a public screen is mostly a public device, while a PDA is mostly a personal device. Thus the PDA interface contains a fully editable personal notes area (Fig. 2, bottom), which has no such counterpart on the public display interface. On the other hand, the public display enhances the presentation and manipulation of publicised notes, a facility that is only weakly shadowed on the PDA. Note that the workstation version (Fig. 9) actually contains versions of both interfaces, as from that user's perspective it is both a personal and public device! Second, we wanted to leverage the input and output capabilities of each device. This is why the public 
display is a shared visual space, where text items (the notes) can be moved around and annotated with drawing marks. In contrast, the PDAs can only show these notes within a scrollable list: we felt that the limited screen space made it inappropriate to retain the spatial information.

Of course, these design differences introduced problems. The obvious one is that items brought back into the PDA's public space did not maintain the spatial relations between notes. The issue is how one deals with information that would be lost when it cannot be presented across devices. A second problem is that our design stressed the use of the PDA as the major place to create notes. In practice, users found that text entry via PDA in the groupware setting was slow, tedious and errorprone. They would much rather have interacted directly with the public display. Unfortunately, the public display had only one mouse/keyboard: it did not allow simultaneous access except through the PDA.

In summary, we believe that devices should be treated as different entities enabling different acts as each affords different powers and capabilities. However, we also recognise that it will be difficult to find a good balance between these devices when used together.

\subsection{Design issues raised by having a rigid notion of public versus private artefacts}

SharedNotes explicitly distinguishes between personal and public notes in many ways. Personal and public notes are placed in separate areas on the PDA; people cannot edit and manipulate them the same way; and so on. This makes a fairly rigid (and perhaps extreme) separation between how personal and public items are treated, which raises a new set of design issues.

\section{Personal notes, once made public, cannot be made personal again.}

Moving from personal to public is a one-way operation. We took this position because of the odd nature of groupware: it is unclear as to what is an original, and what is a copy. Yet this distinction is clear in the physical world. For example, if a person takes a personal sticky note and pastes it onto a whiteboard for others to read, it becomes public. If someone takes it off the whiteboard and puts it into their pocket, it is personal again since it is no longer available to others. Alternately, if someone copies the text of the note onto another sticky and places it in their pocket, there are now two copies: one public (the one on the whiteboard) and one personal (the one in the pocket).

As designers, we had to decide how our electronic artefacts would be treated: as originals being moved, as copies, or as something inbetween. SharedNotes is based on the idea that propagating a note onto the public display moves it there. While people can walk away with a view of it, they no longer have the ability to change it as the original resides elsewhere. If we did allow them to change the note, then we have a problem of what to do when people come back together. We would now have multiple instances of a single item, and problems arise when multiple changes have to be merged. We could have designed the system around the idea that everything on the PDA is just a copy of the public note, but this too introduces complexity when merging versions.

In practice, users did not like the fact that once things were made public they could not be made private again. Various comments stress this. One person criticised SharedNotes by remarking that if he publicised something he later regretted, there was no way of removing it from the public domain. Other participants felt that they should be able to recover things and change their public notes as well as their annotation. Another comment was: "just because you have a thought that you express and that you discussed in a group, it does not mean that it is any less your thought".

\section{Should people have to take explicit action to publicise notes?}

In SharedNotes, individuals have to decide what they want to offer to others and then take explicit action - i.e., checking the publicise box - to publicise a note. This means that people have to decide what they want to keep personal, and what should be made public.

We could have designed SharedNotes so that no explicit publication action was required, where all notes would be publicised as soon as people meet. A personal note would simply be a public note not yet visible to others because the opportunity had not arisen. While this would provide a vastly simpler system, it also implies that people would have no ability to keep things personal, such as items they were not ready to offer to the group. Automatic publication denies users the opportunity to express personal relevancy, and discourages them from using the tool as a 
medium in which to develop ideas not yet ready for dissemination.

\section{Should the system provide personal aspects of public notes?}

We did try to soften the distinction between public and private by allowing public artefacts to contain personal aspects. In SharedNotes, for example, the text of a note is completely public. Once offered to the group, it loses its sense of identity, i.e. its creator no longer has any special claim to it. Thus the text can only be edited in the public forum. However, this leads to the problems described previously. Annotations differ because they do retain their sense of identity, where a single note can have multiple annotations by different people. Consequently, all annotations are in the public view, but individual creators (and only the creators) can modify individual annotations. Thus an annotation is a personal aspect of a public note. Similarly, individual rankings of notes are personal aspects: each individual can alter their ranking, which is reflected in the averaged public view of the rank. While somewhat reasonable in principle, the way annotations are treated led to an overly complex system: consequently annotations were rarely used.

On reflection, the three design questions mentioned above all stem from a single and fundamental problem: SharedNotes has too rigid a notion of what is personal and what is public. In section 2, we explained that people smoothly and easily shift their artefacts from personal to public and the many shades in-between (see also [3]). In contrast, SharedNotes has only a binary notion of public and personal. It forces a model of use onto people that is unnatural and inflexible.

\section{Summary and Next Steps}

This is work in progress. We are trying to understand the distinction between personal and public artefacts and how groupware design can support the way people use them. Our SharedNotes system was created to bring out some of these design issues, and to help us articulate the design space around these types of systems.

There are two specific outcomes from this work. First, we champion the use of devices such as SDG and PDAs as equal partners in the personal/groupware environment. Each device should be designed to maximise its particular strengths and capabilities in ways that make sense to that device, and to handle the exchange and presentation of information between them in appropriate ways. This recommendation mirrors Norman's advocating of information appliances [1]. However, we recognise that it will be difficult to design these devices so that they complement each other well.

Second, we recommend against a rigid notion of personal versus public. Instead, we encourage research into system designs that will let people fluidly shift their artefacts from personal to public and the many gradations between in subtle and lightweight ways. How to do this is not yet clear, but we can learn from the ways people do it in the everyday world [3].

\section{Acknowledgements}

We are thankful to a variety of sponsors. Michael Boyle was funded by a STEP grant. Smart Technologies donated the SmartBoard: it is an eloquent product that creates a single display groupware meeting space. Maurice Sharp of 3Com donated the Palm Pilots used in the study. We also thank the National Sciences and Engineering Research Council of Canada for funding Grouplab and its researchers.

This paper expands our earlier work presented at the Workshop on Handheld CSCW [18].

\section{References}

1. Norman DA. The invisible computer. MIT Press, Cambridge, Mass., 1998

2. Tang JC. Findings from observational studies of collaborative work. International Journal of Man-Machine Studies, $1991 ; 34(2): 143-160$

3. Luff P, Heath C. Mobility in collaboration. In: Proceedings of ACM CSCW'98 Conference on ComputerSupported Cooperative Work, ACM Press, New York, 1998: 305-314

4. Siegel J, Kraut R, Miller M, Kaplan D, Bauer M. Collaborative wearable systems research and evaluation. In: Vicleo Program. Video program: Proceedings of ACM CSCW'96 Conference on Computer-Supported Cooperative Work. ACM Press, New York, 1996:9-10

5. Kraut R, Miller M, Siegel J. Collaboration in performance of physical tasks: effects on outcomes and communication. In: Video Programme: Proceedings of ACM CSCW'96 Conference on Computer-Supported Cooperative Work, ACM Press, New York 1996: 57-66

6. Davis R, Lin J, Brotherton J, Landay J, Price M, Schilit B. A framework for sharing handwritten notes. In: Proceedings of the ACM Symposium on User Interface Software and Technology, ACM Press, New York, 1998: 119-120

7. Minenko V. PalmVNC: virtual network computing client for palm platform. http://www.icsi.berkeley.edu/ minenko/ PalmVNC/, 1998

8. Bharat K, Cardelli L. Migratory applications. In: Proceedings of the ACM Symposium on User Interface Software and Technology. ACM Press, New York, 1995: $133-142$ 
9. Richardson T, Bennett F, Mapp G, Harter A, Hopper A. Teleporting - making applications mobile. In: Video Program. Proceedings of ACM CSCW'94 Conference on Computer-Supported Cooperative Work, ACM Press, New York, 1994: 9-10

10. 3Com Corporation. Palm Pilot Handbook. Part number 423-0210-01C. 1565 Charleston. Rd., Mountain View, Calif. See also www.palm.com, 1994

11. Lotus Notes Inc. Lotus EasySync, version 2.1. Lotus Development Corporation, Cambridge, Mass., 1998

12. Johnson C. The impact of time and place on the operation of mobile computing. In: Proceedings of the HCl'97 Conference on People and Computers XII, 1997: $175-190$

13. Kawachiva $K$, Ishikawa $H$. NaviPoint: an input device for mobile information browsing squeezing, stroking and poking. In: Proceedings of ACM CHI' 98 Conference on Human Factors in Computing Systems, ACM Press, New York. 1998: 1-8

14. Rekimoto J. Pick-and-drop: a direct manipulation technique for multiple computer environments. In Proceedings of the ACM UIST ' 97 Symposium on User Interface
Software and Technology, ACM Press, New York, 1997: 31-39

15. Roseman $\mathrm{M}$, Greenberg $\mathrm{S}$. Building real-time groupware with GroupKit, a groupware toolkit. ACM Transactions on Computer Human Interaction, 1996; 3(1):66-106

16. Smart Technologies, Inc. Calgary, Alberta, Canada. www.smarttech.com

17. Myers B, Stiel $H$, Gargiulo R. Collaboration using multiple PDAs connected to a PC. In: Proceedings of the ACM CSCW '98 Conference on Computer Supported Cooperative Work. ACM Press, New York, 1998

18. Greenberg S, Boyle M. Moving between personal devices and public displays. In: Workshop on Handheld CSCW, held at CSCW'98. Available as Technical Report, Department of Computer Science, University of Karlsruhe, Germany. http://www.teco.edu/hcscw/ program.html, 1998

Correspondence to: S. Greenberg, Department of Computer Science, University of Calgary, Calgary, Alberta T2N 1N4 Canada.Email: saul@cpsc.ucalgary.ca 